\title{
Treatment outcomes of helical tomotherapy for hepatocellular carcinoma in terms of intermediate-dose spillage
}

\author{
Sun Hyun Bae ${ }^{1}$, Kwang Hwan Cho ${ }^{1}$, Young Seok Kim ${ }^{2}$, Sang Gyune Kim ${ }^{2}$, Jeong-Ju Yoo ${ }^{2}$, \\ Jae Myung Lee ${ }^{3}$, Min Hee Lee ${ }^{3}$, Sanghyeok Lim ${ }^{3}$, Jae Hong Jung ${ }^{4}$, Sung Hee Lim ${ }^{5}$ \\ ${ }^{1}$ Department of Radiation Oncology, Soonchunhyang University College of Medicine, Bucheon, Korea; ${ }^{2}$ Division of Gastroenterology and \\ Hepatology, Department of Internal Medicine, Soonchunhyang University College of Medicine, Bucheon, Korea; ${ }^{3}$ Department of Radiology, \\ Soonchunhyang University College of Medicine, Bucheon, Korea; ${ }^{4}$ Department of General Surgery, Soonchunhyang University College of \\ Medicine, Bucheon, Korea; ${ }^{5}$ Division of Hematology-Oncology, Department of Internal Medicine, Soonchunhyang University College of Medicine, \\ Bucheon, Korea \\ Contributions: (I) Conception and design: SH Bae, YS Kim; (II) Administrative support: SH Bae; (III) Provision of study materials or patients: SH \\ Bae, YS Kim, SG Kim; (IV) Collection and assembly of data: SH Bae, KH Cho, JJ Yoo, MH Lee, SH Lim; (V) Data analysis and interpretation: SH \\ Bae, YS Kim, SG Kim, JM Lee, JH Jung, SH Lim; (VI) Manuscript writing: All authors; (VII) Final approval of manuscript: All authors. \\ Correspondence to: Sun Hyun Bae, MD, PhD. Department of Radiation Oncology, Soonchunhyang University College of Medicine, Bucheon, 170 \\ Jomaru-ro, Wongmi-gu, Bucheon-si 14584, Gyeonggi-do, Korea. Email: gurigurihaia@hanmail.net.
}

Background: Although helical tomotherapy (HT) tends to increase intermediate-dose spillage by increasing of low-dose region, this has not been fully determined in the clinical setting. Therefore, we investigated treatment outcomes of HT for hepatocellular carcinoma (HCC) with respect to intermediatedose spillage.

Methods: We retrospectively reviewed 20 HCC patients, who received high-dose radiotherapy (RT) using HT with radical intent between April 2014 and September 2017. In accordance with the Barcelona Clinic Liver Cancer (BCLC) classification, stage was 0 in 7 patients, $\mathrm{A}$ in 3 patients, $\mathrm{B}$ in 5 patients, and $\mathrm{C}$ in 5 patients. Baseline Child-Pugh class was A in 18 patients and $\mathrm{B}$ in 2 patients. The median tumor size was $2.5 \mathrm{~cm}$ (range, 1-11 cm). Helical intensity-modulated radiotherapy (IMRT) technique was applied in all patients: among these, 13 patients were treated with stereotactic body radiotherapy (SBRT). The median fraction size was 12 Gy (range, 2-15 Gy), and the median total dose was 50 Gy (range, 44-60 Gy). Intermediate-dose spillage was assessed by the Radiation Therapy Oncology Group recommendation from 22 HT planning data, as follows: R50\% means the ratio of the $50 \%$ prescription isodose volume to the planning target volume (PTV).

Results: The median follow-up period after HT was 22 months. The local progression-free survival (LPFS) and progression-free survival (PFS) rates were $89 \%$ and $59 \%$ at 1 year, and $82 \%$ and $30 \%$ at 2 years, respectively. The overall survival rate was $100 \%$ at 1 year and $85 \%$ at 2 years, respectively. In terms of intermediate-dose spillage, minor or major deviations were noted in the R50\% of 20 HT plans (91\%). However, 1 patient (5\%) experienced classic radiation-induced liver disease, and severe toxicity $\geq$ grade 3 was not reported.

Conclusions: Although HT for HCC tends to increase intermediate-dose spillage, the treatment results were favorable with that reported in other published studies.

Keywords: Dose constraints; hepatocellular carcinoma (HCC); helical tomotherapy (HT); radiotherapy (RT)

Submitted Sep 17, 2020. Accepted for publication Dec 18, 2020.

doi: $10.21037 /$ tcr-20-2912

View this article at: http://dx.doi.org/10.21037/tcr-20-2912 


\section{Introduction}

Hepatocellular carcinoma (HCC) is the fifth most common cancer and lead to major cancer-related deaths worldwide (1). As this neoplasm develops in patients with cirrhosis and additional comorbidities in most cases, the disease prognosis and the best treatment options may vary depending on the tumor burden, degree of liver dysfunction, and the patients' general conditions (2). Liver resection, liver transplantation, or radiofrequency ablation (RFA), all potential curative therapies for HCC, should be considered as the firstline treatment options when possible (3). However, about $30 \%$ of the patients initially diagnosed with HCC are appropriate for curative therapy. Locoregional treatment modalities, including transarterial chemoembolization (TACE), transarterial radioembolization, and external beam radiotherapy (RT), are considered for patients who are not candidates for curative therapy. Traditionally, RT for HCC has a limited role due to the low tolerance of the whole liver for radiation and the risk of radiation-induced liver disease (RILD), although HCC is a radiation-sensitive tumor (4). With advancements in RT techniques, including development of 3-dimensional conformal radiotherapy (3DCRT), intensity-modulated radiotherapy (IMRT) and stereotactic body radiotherapy (SBRT), the role of RT has expanded from a palliative to a curative intent aim, and treatment result has shown high rates of sustained local control in patients with varying states of $\operatorname{HCC}(5,6)$.

Helical tomotherapy (HT) is a unique rotational IMRT machine using a binary multileaf collimator (MLC) to rapidly open and close the apertures in front of the different beam elements in the fan beam. It uses a slow and continuous movement of the treatment couch with a quickly rotating $\mathrm{X}$-ray source to make many rotations possible in a brief space of time utilizing a helical dose delivery technique analogous to the spiral computed tomography (CT) scanner (7). HT showed a significant improvement in the conformity index (CI) and homogeneity index (HI) for target volume coverage compared with 3DCRT and conventional MLC-mounted linear accelerator-based IMRT in HCC patients (8). However, the mean dose and lowdose region of the normal liver (NL), which is the critical constraint with respect to RT for HCC, increase in HT (9). It is not documented whether the rise in intermediate-dose spillage in the NL may increase the risk of RILD when HT is performed. One study reported the safety of lung SBRT using HT in terms of intermediate-dose spillage (10). Although all RT plans showed major or minor variations in the $\mathrm{R} 50 \%$ [the ratio of the volume of $50 \%$ the prescription dose isodose to the volume of the planning target volume (PTV)], defined by the Radiation Therapy Oncology Group (RTOG) 0915 recommendation, only $8 \%$ of the patients experienced radiation pneumonitis (11).

Therefore, we investigated the treatment outcomes of HT for HCC in terms of intermediate-dose spillage. We present the following article in accordance with the STROBE reporting checklist (available at http://dx.doi. org/10.21037/tcr-20-2912).

\section{Methods}

\section{Patient's selection}

From April 2014 to September 2017, 26 HCC patients received HT for the liver tumor at Soonchunhyang University College of Medicine, Bucheon. We retrospectively reviewed the patients' medical records, and excluded some patients for the following reasons: (I) incomplete HT due to deterioration of ascites during RT (1 patient); (II) transfer to another hospital and loss of follow-up ( 2 patients); and (III) HT with palliative intent (3 patients). The remaining 20 patients, who received high-dose RT using HT with curative intent, were included in the current study. Seven patients were diagnosed with stage 0 according to the Barcelona Clinic Liver Cancer (BCLC) staging system, and they were treated with high-dose RT using HT because of refusal of surgery or inoperable status due to comorbidity. Three patients were classified as stage A and received HT because of abandonment of liver transplantation due to limitation of donor organ or unsuitable location for RFA. Five patients were Stage B and 5 were stage C. All patients underwent TACE, and follow-up CT images presented viable tumor after incomplete TACE. The study was conducted in accordance with the Declaration of Helsinki (as revised in 2013). This retrospective study was approved by the institutional review board (IRB) of Soonchunhyang University College of Medicine, Bucheon (2017-12-002001). Because of the retrospective nature, the requirement of written informed consent was waived.

\section{HT technique}

All patients took planning CT scans with free breathing in the supine position with both arms raised above the head. A posterior vacuum-lock body fixation device was used for immobilization. To reduce respiratory movement, patients 
were requested to take shallow breaths, and an anterior vacuum-sealed cover sheet or belt was applied. Contrastenhanced CT with the helical mode (rotation time: $1 \mathrm{~s}$ ) and subsequent CT scanning (rotation time: $1.5 \mathrm{~s}$ ) were performed with a $3 \mathrm{~mm}$ slice thickness. All CT images were imported to a MIM workstation (MIM Software Inc., Cleveland, OH, USA). Gross tumor volume (GTV) was defined as enhancing lesions, including lipiodol-laden areas, on axial CT images. When necessary, liver dynamic enhanced CT and/or magnetic resonance imaging (MRI) were used to delineate the GTV accurately. The tumor volume used during HT planning was larger than the GTV according to the discretion of the treating radiation oncologist when identifying the borders of the tumor on 2 simulation CT images. This was referred to as the internal target volume (ITV). The PTV was defined as ITV plus asymmetric 3-10 $\mathrm{mm}$ margins in all directions to reduce the RT dose to the stomach, duodenum, intestine, or heart. An additional $2-5 \mathrm{~mm}$ margin was included in the longitudinal direction to compensate for uncertainties resulting from respiratory liver motion.

All structures were transferred to a Tomotherapy Hi Art II Planning System (Accuray Inc., Sunnyvale, CA, USA) for inverse treatment planning. HT plans were created with a pitch of $0.143-0.287$, a modulating factor of 2 or 2.4 , and a longitudinal aperture size of 1 or $2.5 \mathrm{~cm}$. The final dose was calculated through the collapsed cone convolution superposition dose calculation algorithm. All plans were made using the helical-IMRT technique and subdivided into IMRT ( $\leq 7$ Gy) and SBRT (>7 Gy) according to fraction size. SBRT or IMRT was individually applied according to the PTV, NL volume (NLV), and proximity to the gastrointestinal (GI) organ or central biliary tract. In cases with PTV $<1 \mathrm{~cm}$ from the GI organ, we applied simultaneous integrated boost (SIB)-IMRT (12). At least $90 \%$ of the prescription dose should cover the PTV. In terms of liver constraints, at least $700 \mathrm{~mL}$ of the NLV did not receive a total dose $>18$ Gy [reverse $\mathrm{V}_{18 \mathrm{~Gy}}\left(\mathrm{rV}_{18 \mathrm{~Gy}}\right)$, $\geq 700 \mathrm{~mL}$ ] for SBRT, and the mean dose of the NL was $\leq 23 \mathrm{~Gy}$ for IMRT. The maximal dose $\left(\mathrm{D}_{\max }\right)$ for the stomach and duodenum was $\leq 30$ Gy for SBRT and $\leq 44$ Gy for IMRT. The $\mathrm{D}_{\max }$ for the remaining bowel was $\leq 33$ Gy for SBRT and $\leq 60$ Gy for IMRT. We selected the fractionation scheme considering a dose-volume histogram (DVH) for the normal organ. The total doses were converted into biologically equivalent dose (BED) for the equal comparisons of dose effects of various fraction sizes $(\alpha / \beta=10)$.

\section{Failure definition and toxicity assessments}

Regular follow up was undertaken at 1-2 months after the completion of HT and then at 3-month intervals using CT or MRI. Local failure (LF) was defined as either progressive disease according to the Modified Response Evaluation Criteria in Solid Tumors or regrowth in any direction beyond that reported in pre-HT images of the treated lesions. Local-progression-free survival (LPFS) was estimated from start date of HT to the date of LF or last follow-up. Progression-free survival (PFS) and overall survival (OS) was estimated from HT to the date of tumor progression recorded at any site, and the date of death from any cause or last follow-up. Hepatic toxicity was defined as classic RILD (i.e., anicteric hepatomegaly, ascites, or elevated alkaline phosphatase level more than twice the upper limit of the normal value) and non-classic RILD [i.e., elevation of liver transaminases more than 5 times the upper limit of the normal level, or a worsening of the Child-Pugh (CP) score $\geq 2$ points], which occurred within 4 months after HT. Other toxicities were assessed according to the National Cancer Institute Common Terminology Criteria for Adverse Events, version 4.0, and treatment-related severe toxicity was defined as grade $\geq 3$ adverse events or classic/non-classic RILD.

\section{Analysis of the dosimetric parameters and statistics}

DVH analysis was used to assess dosimetric quality. To estimate the PTV coverage, doses at $95 \%$ of the PTV $\left(\mathrm{D}_{95 \%}\right)$ were acquired. The $\mathrm{HI}$ was defined as $\mathrm{D}_{2 \%}$ of the PTV minus $\mathrm{D}_{98 \%}$ of the PTV divided by the prescription dose. CI was defined as the ratio of the prescription isodose volume to the PTV. To estimate high-dose spillage, the percent ratio of the cumulative volume of all tissues outside the PTV receiving a dose $>105 \%$ of the prescription dose to the PTV (\%) was calculated. To estimate intermediate-dose spillage, $\mathrm{R}_{50 \%}$ was calculated. To assess the risk of hepatic toxicity, the mean dose and $\mathrm{rV}_{18 \mathrm{~Gy}}$ of $\mathrm{NL}$ were derived from the DVHs. Individual plans were evaluated using the RTOG 0915 recommendation as the reference points.

\section{Statistical analysis}

The Kaplan-Meier method was used to analyze survivals, and the log-rank test was applied to compare prognostic factors with survivals. All statistical analyses were undertaken using the SPSS software version 14.0 (SPSS Inc., Chicago, IL, USA), 
and a two-sided $\mathrm{P}<0.05$ was considered statistically significant.

\section{Results}

\section{Patient characteristics}

The 20 patients were comprised of 16 males and 4 females, with an age ranging from 50 to 80 years (median, 64 years). Twelve patients (60\%) were infected with hepatitis B virus. Except for 1 patient, all patients received various courses of previous treatments (range, 1-16 courses), including surgery, RFA, TACE, and RT. Baseline CP class was A in 18 patients and $\mathrm{B}$ in 2 patients. Concurrent hepatic arterial infusion chemotherapy was administered in 1 patient during HT. Median tumor size was $2.5 \mathrm{~cm}$. Seventeen patients had single lesion and 3 patients had 2 lesions; 2 patients planned to receive HT for one lesion, in which RT target was partial, followed by TACE for another lesion. Seven patients received IMRT with a fraction size of $2-4.5$ Gy and the total doses of 44-54 Gy/10-27 fractions, and 13 patients received SBRT with a fraction size of 11-15 Gy and the total doses of 44-60 Gy/4 fractions. Patients' characteristics are summarized in Table 1.

\section{Treatment results}

The median follow-up time from the date of HT administration was 22 months (range, 3-43 months). The 1 -year and 2-year LPFS rates were $89 \%$ and $82 \%$, respectively. The 1-year and 2-year PFS rates were 59\% and $30 \%$, respectively. The median OS was not reached, and the 1-year and 2-year OS rates were $100 \%$ and $85 \%$, respectively. Figure 1 shows survival graphs. On univariate analysis, the modified International Union Against Cancer tumor (mUICC-T) stage of 1 and 2, BCLC stage of 0 and A, tumor size $\leq 3 \mathrm{~cm}, \mathrm{PTV} \leq 80 \mathrm{~mL}$, and fraction size $>2.5$ Gy were significantly favorable prognostic factors for LPFS. The mUICC-T stage, BCLC stage, tumor size, PTV, fraction size, and $\mathrm{BED}_{10}$ were significant prognostic factors for PFS. There was no significant prognostic factor for OS (Table 2).

One patient experienced classic RILD at 3 months after HT. Another patient experienced grade 2 rib fracture 16 months after HT, and the pain was relieved with oral medication. There was no severe toxicity above grade 3 .

\section{Analysis of the dosimetric parameters}

The tumor was adjacent to the GI organs in 2 patients, who were treated with IMRT using 20 fractions. To compensate for the unexpectedly large interfractional and intrafractional variation of the GI organ, we conducted a re-plan after 15 fractions. Therefore, a total of $22 \mathrm{HT}$ plans from 20 HCC patients were reviewed. Detailed contents for the dosimetric parameters are summarized in Table 3. The $\mathrm{D}_{\max }$ of the PTV was located within the PTV in all cases. Considering that lower HI values indicate a more homogeneous target dose, SIB-IMRT plans had a more heterogeneous target dose. The CI had minor deviations in 5 plans $(23 \%)$ and major deviations in 3 plans (14\%). Major deviation of the high-dose spillage was occurred in 4 plans, all of which used SIB-IMRT techniques. A total of 20 plans (91\%) had minor or major deviations for R50\% in terms of intermediate-dose spillage. The NL constraints were satisfied for all patients. Classic RILD occurred in a patient with minor deviation for R50\%.

\section{Discussion}

In comparison with other malignancies, various treatment guidelines for the HCC have been published worldwide based on different etiologies, medical insurance systems, and socioeconomic status among regions. Major guidelines, particularly in the West, including the BCLC staging system, suggest RT as an alternative treatment modality or limit RT only for the symptom palliation, or never mentioned RT (13). In real-world clinical practice, however, the University of Michigan reported that both RFA and SBRT are effective local treatment modalities for early stage HCC with 2-year LPFS rates of $80 \%$ and $84 \%$, respectively (14). Two meta-analyses showed that TACE plus RT for unresectable HCC improved tumor response and OS compared with TACE alone $(15,16)$. Based on these clinical evidence, some recently published guidelines, such as the National Cancer Comprehensive Network guideline 2020 version and 2018 Korean Liver Cancer Association-National Cancer Center Korea Practice Guidelines, recommend RT as an equal locoregional modality with ablation or TACE $(17,18)$. Since HT was developed as the first commercial system for planning and delivering IMRT, with the first patient being treated in 2002, the indication for HT has been expanded (19). The whole target is always covered by each beam in 3DCRT; the IMRT covers only a part of the PTV at a certain point using the dynamic delivery modes; thus, the interplay effects induced by the interplay between the interfractional movement of the moving organ and the dynamic dose delivery may result in target under- 
Table 1 Patients' characteristics

\begin{tabular}{|c|c|}
\hline Parameter & Median (range)/No. of pts \\
\hline Age, years & 64 [50-80] \\
\hline \multicolumn{2}{|l|}{ Sex } \\
\hline Male & 16 \\
\hline Female & 4 \\
\hline \multicolumn{2}{|l|}{ ECOG } \\
\hline 1 & 20 \\
\hline \multicolumn{2}{|l|}{ Hepatitis } \\
\hline No & 3 \\
\hline Alcohol & 4 \\
\hline HBV & 12 \\
\hline $\mathrm{HCV}$ & 1 \\
\hline \multicolumn{2}{|l|}{ LC } \\
\hline No & 3 \\
\hline Yes & 17 \\
\hline \multicolumn{2}{|l|}{ Previous treatment } \\
\hline No & 1 \\
\hline Surgery & 3 \\
\hline RFA & 3 (cycles of $1-3$ ) \\
\hline TACE & 18 (cycles of $1-16$ ) \\
\hline RT & 2 \\
\hline \multicolumn{2}{|l|}{ Baseline CP class } \\
\hline$A(5 / 6)$ & $18(10 / 8)$ \\
\hline $\mathrm{B}(7 / 8)$ & $2(1 / 1)$ \\
\hline Baseline AFP, ng/mL & $9.9[1.1-74596]$ \\
\hline \multicolumn{2}{|l|}{ mUICC-T } \\
\hline 1 & 7 \\
\hline 2 & 4 \\
\hline 3 & 8 \\
\hline 4 & 1 \\
\hline \multicolumn{2}{|l|}{ mUICC-N } \\
\hline 0 & 20 \\
\hline \multicolumn{2}{|l|}{ PVTT } \\
\hline No & 16 \\
\hline Yes & 4 \\
\hline
\end{tabular}

Table 1 (continued)
Table 1 (continued)

\begin{tabular}{lc}
\hline Parameter & Median (range)/No. of pts \\
\hline Combined treatment & 19 \\
No & 1 \\
Yes & $2.5[1-11]$ \\
Tumor size, cm & $91.8[14.6-586.3]$ \\
PTV, mL & 18 \\
RT target & 2 \\
All & \\
Partial & 7 \\
RT technique & 13 \\
IMRT & $12[2-15]$ \\
SBRT & $50[44-60]$ \\
Fraction size, Gy & $105.6[53.7-150.0]$ \\
Total dose, Gy & \\
BED &
\end{tabular}

HBV, hepatitis B virus; HCV, hepatitis C virus; RFA, radiofrequency ablation; TACE, transarterial chemoembolization; RT, radiotherapy; CP, Child-Pugh; AFP, $\alpha$-fetoprotein; mUICC, the modified International Union Against Cancer Stage; T, tumor; N, lymph nodes; PVTT, portal vein tumor thrombosis; IMRT, intensity-modulated radiotherapy; SBRT, stereotactic body radiotherapy; $\mathrm{BED}_{10}$, biologically effective dose when $\alpha / \beta$ ratio was assumed to be 10 Gy.

dosing (20). Theoretically, the physical properties of HT, composed of the rapid 360 degree rotating beams and slowly and continuously moving couch, may increase the interplay effects for moving organ compared to LINACbased IMRT. A motion phantom study using HT validated that HT is an effective technique for treating moving tumors and hypofractionation (21). In the clinical setting, HT for 45 unresectable but confined intrahepatic HCC patients, with a median total dose of 54 Gy (2.2-5.5 Gy/ fraction), showed an OS rate of $73 \%$ at 2 years (22). A phase I/II trial using HT-based SBRT for inoperable HCC $\leq 6 \mathrm{~cm}$ showed an LPFS rate of $81 \%$ and an OS rate of $81 \%$ at 2 years (23). The current study shows favorable treatment outcomes with an LPFS rate of $82 \%$ and an OS rate of $85 \%$ at 2 years. These findings suggest that HT for HCC is an effective treatment modality, regardless of the number of fractions.

Radiobiologically, the liver is a typical organ of a parallel 

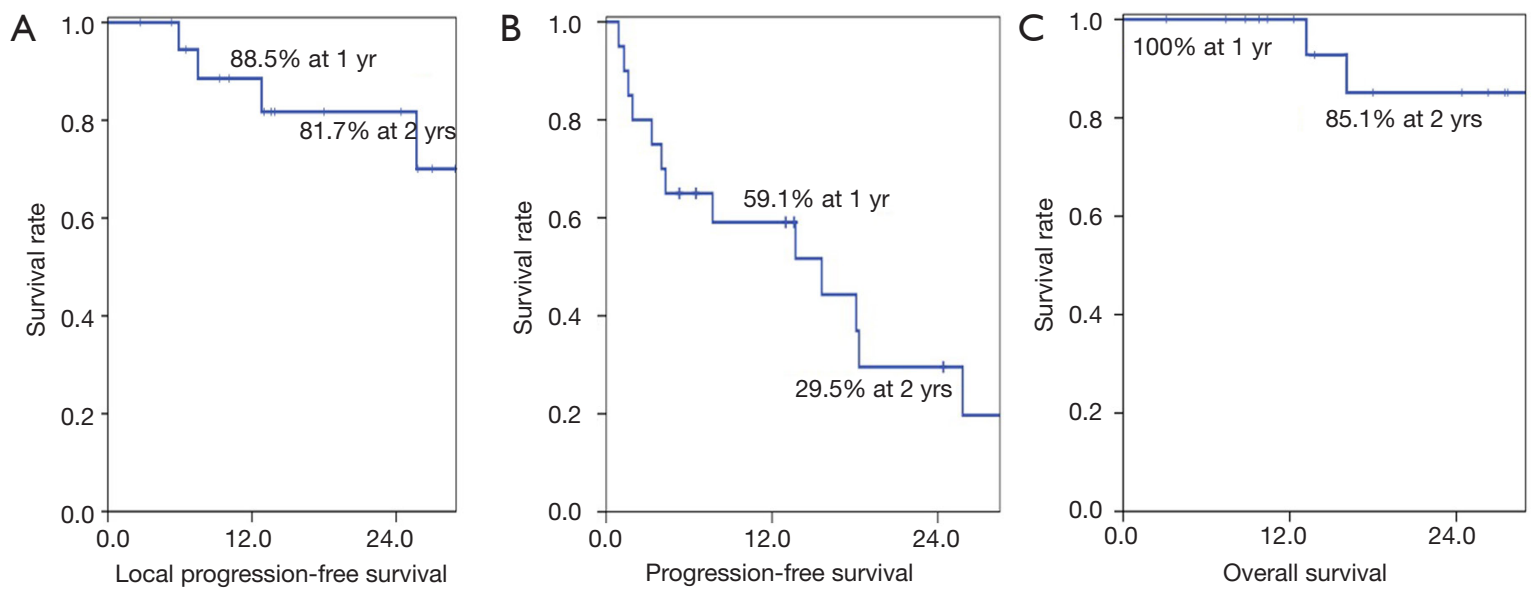

Figure 1 Local progression-free survival (A), progression-free survival (B), and overall survival (C) curves.

Table 2 Univariate analysis for parameters affecting local progression-free survival (LPFS), progression-free survival (PFS), and overall survival (OS)

\begin{tabular}{|c|c|c|c|c|c|c|c|c|}
\hline Parameters & Variable & No. of pts & 2-yr LPFS (\%) & $P$ value & 2-yr PFS (\%) & $P$ value & 2-yr OS (\%) & $P$ value \\
\hline \multirow[t]{2}{*}{ Age, years } & $\leq 60$ & 7 & 83 & 0.447 & 51 & 0.066 & 100 & 0.885 \\
\hline & $>60$ & 13 & 83 & & 15 & & 73 & \\
\hline \multirow[t]{2}{*}{ Sex } & Male & 16 & 77 & 0.322 & 25 & 0.237 & 91 & 0.369 \\
\hline & Female & 4 & 100 & & 50 & & 67 & \\
\hline \multirow[t]{2}{*}{ mUICC-T } & 1,2 & 11 & 100 & 0.007 & 49 & 0.024 & 86 & 0.455 \\
\hline & 3,4 & 9 & 60 & & 11 & & 83 & \\
\hline BCLC stage & $0, A$ & 10 & 100 & 0.012 & 54 & 0.006 & 83 & 0.662 \\
\hline \multirow[t]{2}{*}{ RT technique } & IMRT & 7 & 71 & 0.081 & 0 & 0.057 & 60 & 0.051 \\
\hline & SBRT & 13 & 89 & & 46 & & 100 & \\
\hline \multirow[t]{2}{*}{ Tumor size, cm } & $\leq 3$ & 12 & 100 & 0.005 & 52 & 0.000 & 88 & 0.982 \\
\hline & $>3$ & 8 & 54 & & 0 & & 80 & \\
\hline \multirow[t]{2}{*}{ PTV, mL } & $\leq 80$ & 10 & 100 & 0.011 & 60 & 0.000 & 86 & 0.871 \\
\hline & $>80$ & 10 & 60 & & 0 & & 83 & \\
\hline \multirow[t]{2}{*}{ Fraction size, Gy } & $\leq 2.5$ & 6 & 67 & 0.042 & 0 & 0.019 & 75 & 0.458 \\
\hline & $>2.5$ & 14 & 90 & & 48 & & 89 & \\
\hline
\end{tabular}

CP, Child-Pugh; mUICC, the modified International Union Against Cancer Stage; T, tumor; BCLC, Barcelona Clinic Liver Cancer; PVTT, portal vein tumor thrombosis; RT, radiotherapy; IMRT, intensity-modulated radiotherapy; SBRT, stereotactic body radiotherapy; PTV, planning target volume; $\mathrm{BED}_{10}$, biologically effective dose when $\alpha / \beta$ ratio was assumed to be 10 Gy. 
Table 3 Dosimetric parameters in 22 radiotherapy planning data

\begin{tabular}{|c|c|c|c|c|c|c|c|c|c|c|c|c|c|}
\hline Plan & $\begin{array}{l}\text { PTV } \\
(\mathrm{mL})\end{array}$ & $\begin{array}{l}\text { NLV } \\
(\mathrm{mL})\end{array}$ & $\begin{array}{c}\text { Total, Dose } \\
\text { (Gy) }\end{array}$ & $\begin{array}{c}\text { Fx dose } \\
\text { (Gy) }\end{array}$ & $\begin{array}{l}\text { PTV-D } D_{\max } \\
(\%)\end{array}$ & $\begin{array}{c}\text { PTV-D } \\
\text { (\%) }\end{array}$ & $\mathrm{HI}$ & $\mathrm{Cl}$ & $\begin{array}{l}\text { HDS } \\
(\%)\end{array}$ & $\mathrm{R} 50 \%$ & $\begin{array}{l}\text { NL-mean, } \\
\text { dose (Gy) }\end{array}$ & $\begin{array}{l}\text { rNL-18Gy } \\
\quad(m L)\end{array}$ & $\begin{array}{c}\text { NL-V30Gy } \\
(\%)\end{array}$ \\
\hline IMRT & 60 & 699 & 45 & 4.5 & 117.5 & 102.4 & 0.1 & $1.2^{\mathrm{a}}$ & 5.8 & 4.2 & 12.3 & 574 & 7.0 \\
\hline IMRT & 135 & 1,371 & 54 & 2 & 105.4 & 99.7 & 0.0 & 0.9 & 0.0 & $3.6^{\mathrm{a}}$ & 13.5 & 954 & 10.0 \\
\hline IMRT & 147 & 987 & 50 & $3 / 2$ & 164.8 & 102.0 & 0.6 & $2.1^{b}$ & $93.3^{b}$ & $7.9^{b}$ & 23.3 & 512 & 29.8 \\
\hline IMRT & 410 & 1,117 & 37.5 & $3.5 / 2.5$ & 154.3 & 104.9 & 0.5 & $1.9^{b}$ & $74.9^{b}$ & $5.9^{b}$ & \multirow{2}{*}{$20.8^{c}$} & \multirow{2}{*}{$686^{c}$} & \multirow{2}{*}{$21.7^{c}$} \\
\hline IMRT & 401 & 1,099 & 11 & $3.5 / 2.2$ & 177.9 & 105.1 & 0.7 & $2.2^{\mathrm{b}}$ & $106.7^{b}$ & $7.2^{\mathrm{b}}$ & & & \\
\hline IMRT & 581 & 1,036 & 33 & $3 / 2.2$ & 150.5 & 103.5 & 0.5 & 1.1 & 5.9 & $4.1^{\mathrm{b}}$ & $18.9^{d}$ & $637^{d}$ & $23.3^{d}$ \\
\hline SBRT & 28 & 1,360 & 60 & 15 & 104.1 & 100.0 & 0.0 & 1.1 & 0.0 & $4.8^{a}$ & 11.1 & 1,079 & 6.9 \\
\hline SBRT & 29 & 812 & 52 & 13 & 106.1 & 100.0 & 0.0 & $1.2^{\mathrm{a}}$ & 0.0 & $5.0^{\mathrm{a}}$ & 7.4 & 715 & 6.2 \\
\hline SBRT & 44 & 1,762 & 60 & 15 & 104.0 & 99.7 & 0.0 & 1.0 & 0.0 & $5.1^{a}$ & 4.8 & 1,595 & 4.5 \\
\hline SBRT & 50 & 909 & 48 & 12 & 105.7 & 98.4 & 0.1 & 0.9 & 0.0 & 3.6 & 10.9 & 762 & 5.7 \\
\hline SBRT & 57 & 1,502 & 52 & 13 & 105.1 & 99.8 & 0.0 & 1.0 & 0.0 & $4.5^{\mathrm{a}}$ & 9.4 & 1,314 & 3.6 \\
\hline $\mathrm{SBRT}^{\mathrm{e}}$ & 72 & 1,197 & 52 & 13 & 110.8 & 100.7 & 0.1 & 1.1 & 0.1 & $4.3^{\mathrm{a}}$ & 11.2 & 971 & 7.1 \\
\hline SBRT & 80 & 974 & 48 & 12 & 107.5 & 100.7 & 0.1 & 1.1 & 0.0 & $4.6^{\mathrm{a}}$ & 10.6 & 773 & 8.0 \\
\hline
\end{tabular}

NLV, normal liver volume; $\mathrm{HI}$, homogeneity index $\left(\mathrm{D}_{2 \%}\right.$ of the PTV minus $\mathrm{D}_{98 \%}$ of the PTV divided by the prescription dose); Cl, conformity index $(\mathrm{Cl})$, defined as the ratio of the prescription isodose volume to the PTV; HDS, high dose spillage (cumulative volume of all tissue outside PTV receiving a dose $>105 \%$ of the prescription dose; R50\%, the ratio of the $50 \%$ prescription isodose volume to the PTV; rNL18Gy, NLV receiving <18 Gy; NL-V30Gy, NLV receiving 30 Gy. ${ }^{\text {a }}$, minor deviation according to RTOG 0915 protocol; ${ }^{\text {, }}$, major deviation according to RTOG 0915 protocol; ${ }^{c, d}$, Two patients changed treatment plan during HT. Therefore, we divided dosimetric parameters for PVT but summarized dosimetric parameters for normal liver as organ at risk; ${ }^{e}$, dosimetric parameter for a patient with classic RILD.

architecture model. Although RILD mostly occurs if the critical volume of the NL damaged is over a threshold, the risk may be additionally increased by dose distribution of the functional reserve and subunit radiosensitivity (24). HT achieves better $\mathrm{CI}$ and $\mathrm{HI}$ than 3DCRT at the expense of a greater low-dose bath (9). This low-dose bath may impact the partial volume tolerance of the NL and may increase the risk of RILD even if the same mean dose of the NL is irradiated. At present, with modern RT technology, experts recommend that attention must be paid to the specific isodose distribution, including that for intermediate doses such as 20-30 Gy, during treatment planning evaluation (25). However, the tolerance of the NL by intermediate-dose spillage from HT has never been clinically validated despite many dosimetric studies. We could find a clue from the lung, another typical organ of a parallel architectural model. One institution began lung SBRT with HT since 2008 and applied constraints from the RTOG 0915 recommendation (10). They noticed that many SBRT plans with HT for the lung did not meet R50\% and assessed the clinical validity of 
R50\% in 74 patients (81 lesions and 79 plans). All plans had major deviations $(39 \%)$ or minor deviations $(61 \%)$ from R50\%. However, treatment-related toxicity was minimal: 2 patients (3\%) experienced chest wall pain; 6 patients (8\%) experienced radiation pneumonitis (grade $1-2$ in 4 patients, grade 3 in 1 patient, and grade 5 in 1 patient). One patient with grade 5 radiation pneumonitis underwent multiple lung surgeries and high-dose RT for lung cancer before SBRT, and fatal toxicity might be induced from surgeries and underlying poor lung function. The authors concluded that HT is a safe SBRT modality for lung cancer, despite not being able to meet R50\%. Our study also showed that 20 HT plans had minor deviations (41\%) or major deviations $(50 \%)$ from R50\%, although all patients met the NL constraints. Among these, only 1 patient with minor deviation of R50\% experienced classic RILD and subsequently recovered. This suggests that the increase in intermediate-dose spillage from a greater low-dose bath by HT is not related to the increase of hepatic toxicity if the NL constraints are met, and that HT for HCC is a safe treatment modality.

There were several limitations in this study. First, we applied R50\% for both SBRT and IMRT cases. Actually this constraint was made for measurement of the steepness of the dose gradient for SBRT. Considering that IMRT is used for large-sized HCC or HCC located near GI organ or central biliary tract, R50\% for IMRT does not reflect intermediate-dose spillage, although linear interpolation is permitted for unspecified values of PTVs. However, this might be the best method because R50\% by the RTOG recommendation is the only accepted value in the world to assess intermediate-dose spillage. Second, this study was a retrospective analysis with a small sample size. Therefore, selection bias may arise, and the rate of treatment-related toxicity may be underestimated. Although we found that deviation of $\mathrm{R} 50 \%$ from the desired value did not increase the risk of hepatic toxicity, further studies based on a large population will be needed to confirm the result.

In conclusion, the current study reported an LPFS rate of $82 \%$ and an OS rate of $85 \%$ at 2 years, respectively, using HT. These treatment results are comparable with those published in other published studies using different RT machines, and support that HT is an effective treatment modality. In terms of intermediate-dose spillage, minor or major deviations from the desired $\mathrm{R} 50 \%$ were noted in $91 \%$ of HT plans. However, the only 1 patient $(5 \%)$ experienced classic RILD and this supports that HT is a safe treatment modality. Although this is the first study to validate the clinical significance of unmet $\mathrm{R} 50 \%$ by $\mathrm{HT}$ for the treatment of HCC, additional studies based on larger populations will be needed.

\section{Acknowledgments}

Funding: This work was supported by the Soonchunhyang University Research Fund. No funding bodies had any role in study design, data collection and analysis, decision to publish, or preparation of the manuscript.

\section{Footnote}

Reporting Checklist: The authors have completed the STROBE reporting checklist. Available at http://dx.doi. org/10.21037/tcr-20-2912

Data Sharing Statement: Available at http://dx.doi. org/10.21037/tcr-20-2912

Conflicts of Interest: All authors have completed the ICMJE uniform disclosure form (available at http://dx.doi. org/10.21037/tcr-20-2912). All authors have no conflicts of interest to declare.

Ethical statement: The authors are accountable for all aspects of the work in ensuring that questions related to the accuracy or integrity of any part of the work are appropriately investigated and resolved. The study was conducted in accordance with the Declaration of Helsinki (as revised in 2013). This retrospective study was approved by the institutional review board (IRB) of Soonchunhyang University College of Medicine, Bucheon (2017-12-002001). Because of the retrospective nature, the requirement of written informed consent was waived.

Open Access Statement: This is an Open Access article distributed in accordance with the Creative Commons Attribution-NonCommercial-NoDerivs 4.0 International License (CC BY-NC-ND 4.0), which permits the noncommercial replication and distribution of the article with the strict proviso that no changes or edits are made and the original work is properly cited (including links to both the formal publication through the relevant DOI and the license). See: https://creativecommons.org/ licenses/by-nc-nd/4.0/. 


\section{References}

1. Global Burden of Disease Liver Cancer Collaboration, Akinyemiju T, Abera S, et al. The Burden of Primary Liver Cancer and Underlying Etiologies From 1990 to 2015 at the Global, Regional, and National Level: Results From the Global Burden of Disease Study 2015. JAMA Oncol 2017;3:1683-91.

2. Vitale A, Trevisani F, Farinati F, et al. Treatment of hepatocellular carcinoma in the Precision Medicine era: from treatment stage migration to therapeutic hierarchy. Hepatology 2020;72:2206-18.

3. Couri T, Pillai A. Goals and targets for personalized therapy for HCC. Hepatol Int 2019;13:125-37.

4. Emami B, Lyman J, Brown A, et al. Tolerance of normal tissue to therapeutic irradiation. Int J Radiat Oncol Biol Phys 1991;21:109-22.

5. Dawson LA, Ten Haken RK, Lawrence TS. Partial irradiation of the liver. Semin Radiat Oncol 2001;11:240-6.

6. Bang A, Dawson LA. Radiotherapy for HCC: Ready for prime time? JHEP Rep 2019;1:131-7.

7. Galvin JM, De Neve W. Intensity modulating and other radiation therapy devices for dose painting. J Clin Oncol 2007;25:924-30.

8. Lee IJ, Seong J, Koom WS, et al. Selection of the optimal radiotherapy technique for locally advanced hepatocellular carcinoma. Jpn J Clin Oncol 2011;41:882-9.

9. Bae SH, Jang WI, Park HC. Intensity-modulated radiotherapy for hepatocellular carcinoma: dosimetric and clinical results. Oncotarget 2017;8:59965-76.

10. Amin NP, Nalichowski A, Campbell S, et al. Helical Therapy is Safe for Lung Stereotactic Body Radiation Therapy Despite Limitations in Achieving Sharp Dose Gradients. Technol Cancer Res Treat 2017;16:1173-8.

11. Videtic GM, Hu C, Singh AK, et al. Erratum. A randomized phase 2 study comparing 2 stereotactic body radiation therapy schedules for medically inoperable patients with stage I peripheral non-small cell lung cancer: NRG Oncology RTOG 0915 (NCCTG N0927). Int J Radiat Oncol Biol Phys 2016;94:638.

12. Kim TH, Park JW, Kim YJ, et al. Simultaneous integrated boost-intensity modulated radiation therapy for inoperable hepatocellular carcinoma. Strahlenther Onkol 2014;190:882-90.

13. Rim CH, Cheng J, Huang WY, et al. An evaluation of hepatocellular carcinoma practice guidelines from a radiation oncology perspective. Radiother Oncol 2020;148:73-81.

14. Wahl DR, Stenmark MH, Tao Y, et al. Outcomes After Stereotactic Body Radiotherapy or Radiofrequency Ablation for Hepatocellular Carcinoma. J Clin Oncol 2016;34:452-9.

15. Meng MB, Cui YL, Lu Y, et al. Transcatheter arterial chemoembolization in combination with radiotherapy for unresectable hepatocellular carcinoma: a systematic review and meta-analysis. Radiother Oncol 2009;92:184-94.

16. Huo YR, Eslick GD. Transcatheter Arterial Chemoembolization Plus Radiotherapy Compared With Chemoembolization Alone for Hepatocellular Carcinoma: A Systematic Review and Meta-analysis. JAMA Oncol 2015;1:756-65.

17. National Comprehensive Cancer Network (NCCN)®. NCCN Guidelines version 3. 2020. Available online: https://www.nccn.org/professionals/physician_gls/pdf/ hepatobiliary.pdf

18. Korean Liver Cancer Association; National Cancer Center. 2018 Korean Liver Cancer Association-National Cancer Center Korea Practice Guidelines for the Management of Hepatocellular Carcinoma. Gut Liver 2019;13:227-99.

19. Cho B. Intensity-modulated radiation therapy: a review with a physics perspective. Radiat Oncol J 2018;36:1-10.

20. Zhu Z, Fu X. The radiation techniques of tomotherapy $\&$ intensity-modulated radiation therapy applied to lung cancer. Transl Lung Cancer Res 2015;4:265-74.

21. Kanagaki B, Read PW, Molloy JA, et al. A motion phantom study on helical tomotherapy: the dosimetric impacts of delivery technique and motion. Phys Med Biol 2007;52:243-55.

22. Jiang T, Zeng ZC, Yang P, et al. Exploration of Superior Modality: Safety and Efficacy of Hypofractioned ImageGuided Intensity Modulated Radiation Therapy in Patients with Unresectable but Confined Intrahepatic Hepatocellular Carcinoma. Can J Gastroenterol Hepatol 2017;2017:6267981.

23. Kim JW, Kim DY, Han KH, et al. Phase I/II trial of helical IMRT-based stereotactic body radiotherapy for hepatocellular carcinoma. Dig Liver Dis 2019;51:445-51.

24. Jackson A, Ten Haken RK, Robertson JM, et al. Analysis 
of clinical complication data for radiation hepatitis using a parallel architecture model. Int J Radiat Oncol Biol Phys 1995;31:883-91.

Cite this article as: Bae SH, Cho KH, Kim YS, Kim SG, Yoo JJ, Lee JM, Lee MH, Lim S, Jung JH, Lim SH. Treatment outcomes of helical tomotherapy for hepatocellular carcinoma in terms of intermediate-dose spillage. Transl Cancer Res 2021;10(3):1420-1429. doi: 10.21037/tcr-20-2912
25. Koay EJ, Owen D, Das P. Radiation-Induced Liver Disease and Modern Radiotherapy. Semin Radiat Oncol 2018;28:321-31. 